\title{
A documentação clínica em Terapia Ocupacional: revisáo de literatura
}

\author{
Carla Simon Benevides Panzeri ${ }^{a}$, Marina Silveira Palhares ${ }^{b}$ \\ ${ }^{a}$ Mestre em Terapia Ocupacional, Programa de Pós-graduação em Terapia Ocupacional - PPGTO, Universidade \\ Federal de São Carlos - UFSCar, São Carlos, SP, Brasil

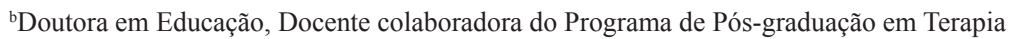 \\ Ocupacional - PPGTO, Universidade Federal de São Carlos - UFSCar, São Carlos, SP, Brasil
}

\begin{abstract}
Resumo: A documentação clínica em Terapia Ocupacional compreende toda informação registrada sobre um cliente/ paciente/usuário, desde o encaminhamento até a alta, e poderá integrar o prontuário do paciente/cliente/usuário ou servir de apoio para a prática exclusiva da Terapia Ocupacional. No Brasil foi verificado que, apesar de ser uma prática profissional obrigatória e rotineira, pouco material técnico-científico sobre o tema está disponível para suporte aos profissionais. Este estudo teve por objetivo identificar, através de revisão de literatura, informações para maior conhecimento e suporte para a prática da documentação clínica por terapeutas ocupacionais. As informações foram analisadas e apresentadas segundo os diferentes temas identificados, buscando pontos em comuns, complementares e divergentes sobre cada um deles. Doze artigos fizeram parte do estudo. As informações obtidas abordavam os temas: métodos de registro, percepção e atitudes dos profissionais, comunicação e linguagem e qualidade. Foi possível realizar uma primeira aproximação com o tema e ter um panorama geral dos aspectos relacionados à documentação clínica em Terapia Ocupacional, no entanto apresentam-se diversas lacunas e as informações mostram-se bastante segmentadas.
\end{abstract}

Palavras-chave: Terapia Ocupacional, Prontuários, Revisão Sistemática.

\section{Occupational Therapy clinical documentation: a literature review}

\begin{abstract}
Occupational therapy clinical documentation comprises all recorded information about a client/patient/ user, from forwarding to discharge, and it may integrate the patient's record or serve as support for the exclusive practice of occupational therapy. In Brazil, despite being a mandatory and routine practice, little scientific-technical material on the subject is available for the support of professionals. This study aimed to identify, through literature review, information for greater understanding and support to the practice of professional activity. The data were analyzed and presented according to the different themes identified, seeking common, complementary and divergent points on each theme. Twelve articles were included in the study. The information obtained addressed themes such as registration methods, perception and attitudes of professionals, communication and language, quality, and others. It was possible to achieve a preliminary approach to the subject and have an overview of aspects related to occupational therapy clinical documentation, but some segmented information and several weaknesses were found.
\end{abstract}

Keywords: Occupational Therapy, Records, Systematic Review.

Autor para correspondência: Carla Simon Benevides Panzeri, Universidade Federal de São Carlos, Alameda das Cerejeiras, 28,Cond. Portal de Itu, CEP 13301-634, Itu, SP, Brasil, e-mail: carlasbpanzeri@gmail.com

Recebido em 21/11/2012; $1^{\text {a }}$ Revisão em 4/6/2013; Aceito em 1/7/2013. 


\section{Introdução}

A documentação clínica em Terapia Ocupacional compreende toda informação registrada sobre um cliente/paciente/usuário, desde o seu encaminhamento até a alta do serviço (MATTHEWS; JABRI, 2004). As informaçôes registradas incluem o raciocínio sobre o diagnóstico, planos de tratamento, ações e resultados da intervenção terapêutica ocupacional (ROGERS; HOLM, 1991). Tais registros podem integrar o prontuário do paciente/cliente/usuário ou servir de apoio para a prática exclusiva da Terapia Ocupacional (MATTHEWS; JABRI, 2004).

$\mathrm{O}$ prontuário do paciente/cliente/usuário é constituído por todo o acervo documental padronizado, organizado e conciso referente ao registro dos cuidados a ele prestados e pelos demais documentos pertinentes à sua assistência (POSSARI, 2007). Mezzomo (1991) ressalta que as informaçóes e documentos contidos no prontuário são elaborados por diferentes autores, o conjunto de profissionais que atendem o paciente/cliente/usuário, incluindo o terapeuta ocupacional.

No desenvolvimento dos registros contidos no prontuário, diversos aspectos estão envolvidos, entre eles: linguagem e comunicação, questôes ético-legais, formas de registro e armazenagem e avaliação da qualidade e auditorias (MATTHEWS; JABRI, 2004; MEZZOMO, 1991; SAMES, 2005).

As informações contidas no prontuário do paciente/cliente/usuário irão servir de apoio à assistência, à pesquisa, à formação profissional, à gestão dos serviços, à gestão pública e aos aspectos ético-legais (FEARING, 1993; MASSAD; MARIN; AZEVEDO NETO, 2003; NOVAES, 2003; POSSARI, 2007; VASCONCELLOS; GRIBEL; MORAES, 2008). Além disso, Stumpf e Freitas (1997) ressaltam a necessidade de se ter informaçóes de qualidade no prontuário para que se alcance o correto atendimento de suas funções.

Matthews e Jabri (2004, p. 99) destacam que "a documentação é a via principal por onde os provedores de saúde comunicam a outros informaçóes sobre um cliente ou paciente".

Atualmente o paciente/cliente/usuário é tido como o provedor de todas as informaçôes constantes no prontuário e como o principal beneficiário dele (POSSARI, 2007), sendo considerado seu proprietário de fato e as instituiçôes, seus guardiōes (REIS et al., 2009). Não obstante, são considerados usuários dos prontuários os próprios pacientes/ clientes/usuários, além de seus familiares e dos profissionais diretamente envolvidos com os cuidados e a gestão e os docentes e alunos de cursos de saúde (BART et al., 2011; VASCONCELLOS; GRIBEL; MORAES, 2008), sendo que a documentação nele contida possibilita a defesa e proteçáo de todos os usuários envolvidos (OLIVEIRA et al., 2012).

Em relação especificamente ao terapeuta ocupacional, Sames (2005) informa que esse profissional pode atuar com uma variedade de clientes e contextos de atendimento, mas em todos eles precisará documentar suas intervenções. No Brasil, a resolução COFFITO 415/2012 (CONSELHO..., 2012) torna obrigatório o registro em prontuário pelo terapeuta ocupacional. Esse documento contém orientações sobre as informaçóes mínimas a constarem no prontuário, sendo enfatizada a necessidade de registro de cada atendimento realizado e a identificação do profissional responsável. Também apresenta orientaçóes sobre a guarda e descarte dos prontuários, além de reforçar que o prontuário pertence ao paciente/cliente/usuário, devendo o terapeuta ocupacional ou a instituição onde ele atua serem seus guardiōes, com o dever de manter sigilo sobre as informações nele contidas.

A Associação Americana de Terapia Ocupacional - AOTA (AMERICAN..., 2008a) apresenta diversas publicaçôes que orientam a prática da documentação clínica. $\mathrm{Na}$ referência mais atual: Diretrizes para a Documentação em Terapia Ocupacional (tradução nossa), publicada em 2008, os registros são descritos segundo três áreas do processo terapêutico: avaliação, intervenção e resultados. Em relação à avaliação inclui registros referentes à triagem e aos relatórios de avaliação inicial e reavaliaçáo periódica. Em relação à intervenção inclui plano de intervenção, notas de contato do serviço de Terapia Ocupacional, relatório de progresso e plano de transição (realizado quando o cliente precisa ser transferido para outro serviço de Terapia Ocupacional). Em relação aos resultados inclui o relatório de alta. Para cada tipo de registro, recomenda quais informaçóes são pertinentes e fundamentais para sua elaboraçáo com qualidade (AMERICAN..., 2008a).

No Brasil foi verificado que, apesar de ser uma prática profissional obrigatória e rotineira, pouco material técnico-científico sobre o tema está disponível para suporte aos profissionais.

Dessa forma, este estudo teve por objetivo realizar revisão de literatura sobre o tema documentação clínica em Terapia Ocupacional visando identificar informaçóes para maior conhecimento e suporte para a prática dessa atividade profissional. Além disso, visava fornecer embasamento para elaboração de projeto de pesquisa de mestrado intitulado "A 
documentação clínica ambulatorial sob a ótica de terapeutas ocupacionais".

Os espaços institucionais ou contextos de atendimento onde o terapeuta ocupacional está inserido podem ser espaços de intervenção em saúde, educação e programas sociais e também o ambiente doméstico e de trabalho (SOARES, 2007). Parte-se, neste estudo, da perspectiva de documentação a partir do campo de assistência em saúde clínico. No entanto, cabe ressaltar que esses profissionais desenvolvem registros também sob diversas outras perspectivas, de outros campos de atuação, como da educação, da assistência social e da cultura e lazer, cujos aspectos relacionados à documentaçáo não serão abordados no presente trabalho.

\section{Método}

Trata-se de um estudo de revisão de literatura, desenvolvida no período de março de 2011 a março de 2012, para identificar produçôes científicas sobre a temática da documentação clínica especificamente para a Terapia Ocupacional.

O desenho de pesquisa adotado pode ser caracterizado como do modelo de revisão de literatura overview, descrito por Grant e Bootht (2009) como aquele que busca trazer um resumo da literatura sobre um determinado tema descrevendo suas características. Seus resultados são normalmente apresentados de forma narrativa, mas podem-se utilizar recursos tabulares e a análise pode ser cronológica, conceitual, temática ou outra. Os autores apontam como ponto forte que a síntese apresentada pode ser bastante ampla e abrangente, sendo valorizada para o estudo de temas vistos pela primeira vez. Apontam como fraqueza metodológica o menor rigor e qualidade em relação a outros tipos de revisão, que fazem uso de métodos mais sistemáticos de busca. Esse modelo de revisão foi adotado devido ao número escasso de textos sobre o tema previamente identificados em bases de dados indexadas, constituindo, portanto, um panorama, uma visão geral do tema.

Dessa forma, através do portal da Biblioteca Virtual em Saúde, foi realizada pesquisa nos bancos de dados: LILACS, IBECS, MEDLINE, Biblioteca Cochrane e SciELO. Também foi realizada busca no sítio eletrônico das bases de dados Google Schoolar e em uma base de dados específica de Terapia Ocupacional, o OTSeeker DataBase. Além disso, foi realizada busca diretamente em sítios eletrônicos de revistas nacionais especializadas de Terapia
Ocupacional: Cadernos de Terapia Ocupacional da UFSCar e Revista de Terapia Ocupacional da USP.

Para a busca foram utilizados os descritores contidos na lista dos Descritores em Ciências da Saúde, DeCs 2011: documentação, registros médicos (recomendados como alternativa para prontuários) e Terapia Ocupacional. Para a busca em língua inglesa foram utilizados os descritores: occupational therapy, documentation, clinical documentation e records.

Para o presente estudo, como critérios de inclusão foram eleitos artigos de qualquer natureza, que abordassem diretamente o tema, publicados em língua portuguesa, inglesa ou espanhola, independentemente do ano de publicação (devido ao pequeno número localizado). Foram excluídos os artigos que abordavam a documentação clínica de forma indireta ou como fonte de dados e náo como objeto de estudo e os publicados em demais línguas.

Após a busca foi realizada a leitura dos resumos, para seleção daqueles com o perfil do estudo. Em seguida foi realizada a tentativa de recuperação na íntegra dos artigos selecionados (por meio digital e em acervo de bibliotecas). Aqueles recuperados na íntegra foram lidos, sendo utilizados neste estudo os que atendiam aos critérios de inclusão.

Para melhor apreensão e aprofundamento da discussão, a análise foi realizada segundo os diferentes temas identificados, buscando pontos em comuns, complementares e divergentes sobre cada um deles. Os temas identificados foram: métodos de registro; percepção e atitudes dos profissionais; comunicação e linguagem; e qualidade.

De 43 artigos previamente selecionados a partir da leitura dos resumos, 12 compuseram a amostra final por atenderem aos critérios de inclusão estabelecidos para esta revisão.

\section{Resultados e discussão}

Conforme apresentado na Tabela 1, observou-se que todos os artigos que compuseram este estudo são estrangeiros, publicados em periódicos de língua inglesa, majoritariamente específicos de Terapia Ocupacional, com maior concentração de publicaçáo no período de 1990 a 1999. O veículo com maior número de publicações foi o Canadian Journal of Occupational Therapy, com quatro artigos. A maior parte dos artigos, seis, é do tipo artigo de pesquisa, três são relatos de experiência e três são comunicaçôes livres.

Em relação ao conteúdo apresentado, segue a discussão conforme os principais diferentes temas identificados. 


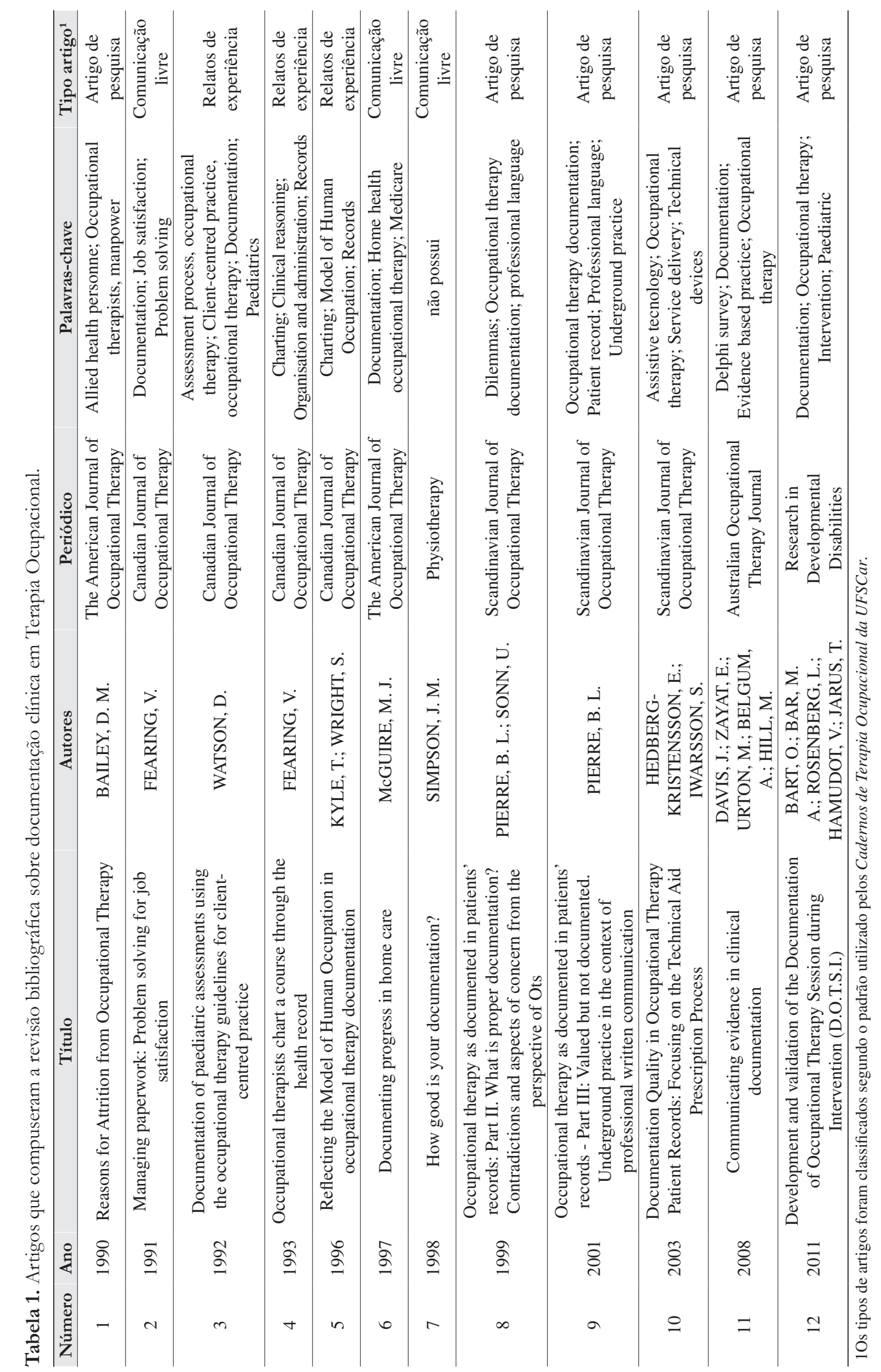




\subsection{Métodos de registro}

Para documentação das intervençôes da Terapia Ocupacional não há um método exclusivo ou padrão, porém, independentemente do método adotado, é fundamental que as informaçôes sejam claras, concisas, objetivas e completas (MATTHEWS; JABRI, 2004). Radomski (2005) enfatiza que ao realizá-la, deve-se considerar o público alvo a quem esse registro se destina.

Na presente revisão, quatro estudos abordando o desenvolvimento de modelos específicos para registro de intervençôes terapêuticas ocupacionais foram identificados, tendo cada um deles adotado referencial teórico distinto para embasamento das propostas desenvolvidas.

O primeiro, de Watson (1992), apresentou um formato para a documentação de relatórios de avaliação desenvolvido em um serviço pediátrico, mas que pode ser utilizado com outras faixas etárias e em diferentes áreas de atuação. Em 1990, os terapeutas ocupacionais da Sociedade Arbutus para Crianças estabeleceram uma comissão para revisão da documentação por eles utilizada e desenvolvimento de um formato de relatório de avaliação que melhor refletisse os referenciais teóricos adotados e os serviços oferecidos. A proposta foi desenvolver um método que refletisse o Modelo Canadense de Desempenho Ocupacional, usando como embasamento teórico para sua proposição tanto as Diretrizes para Terapia Ocupacional Centrada no Cliente quanto a Terminologia Uniforme da Terapia Ocupacional da AOTA. O formato apresentou as informaçôes a constar, a sua organização e a terminologia apropriada, divididas em cinco seçóes: introdução, avaliação, interpretação, objetivos e recomendação. Depois de estabelecido um formato inicial pela comissão, deu-se início ao treinamento dos 21 terapeutas ocupacionais que atuavam na Sociedade, distribuídos em três unidades distintas, atendendo a crianças com dificuldades em diferentes graus, tanto físicas quanto psíquicas, em regime de internação, ambulatorial e programas comunitários. Por dois meses realizaram-se reuniôes semanais para treinamento e revisão sobre como a documentação podia refletir a prática centrada no cliente. $\mathrm{O}$ formato foi aplicado nos diferentes serviços e foi estabelecido um sistema de avaliação por pares para o seu ajuste. Após um ano de uso, concluiu-se que o formato proposto produziu relatórios de avaliação compreensivos, que permitiram registrar a especificidade da Terapia Ocupacional e auxiliar no acompanhamento do progresso da intervenção, além de agilizar possíveis encaminhamentos.
Fearing (1993) apresentou uma proposta para a documentação de todo o processo terapêutico desenvolvido em um hospital universitário canadense baseado nas Diretrizes para Terapia Ocupacional Centrada no Cliente. A proposta é mais abrangente e está intrinsecamente relacionada à intervenção a partir do modelo teórico adotado, considerando a documentação clínica como parte integrante do processo terapêutico. Para seu desenvolvimento utilizou-se o método de "nomeação do problema". Assim, para o registro é dada ênfase ao protagonismo do cliente, sendo que cada problema é identificado, nomeado e validado junto com ele, assim como são negociados as responsabilidades e resultados esperados. Apresentam-se nessa proposta orientaçóes para o registro de cada etapa do processo terapêutico, sendo os registros desenvolvidos como parte do raciocínio clínico e, ao final do tratamento, espera-se que o cliente tenha acesso a todo seu registro clínico e que o leia sem surpresas. Esse modelo esteve em uso por alguns anos em um hospital universitário e passou a ser utilizado por diversas instituiçóes em British Columbia, Canadá, e como base para ensino profissional na University of British Columbia. Concluiu-se que foram identificados diversos benefícios com seu uso, sem especificar-se a metodologia de obtenção de tais dados, entre eles: melhorar o foco de atuação na performance ocupacional, facilitar o entendimento pela equipe do propósito da Terapia Ocupacional e refletir seu raciocínio clínico, e promover consistência na qualidade e conteúdo da documentação, independente do terapeuta ocupacional que a realizou.

Kyle e Wright (1996) apresentaram outro modelo para registro de avaliação, o Screening Assessment Form (SAF) desenvolvido pelos terapeutas ocupacionais de um hospital canadense, o Ottawa Civic Hospital, na área de saúde mental, sendo indicada sua aplicaçáo em outras áreas, com as adaptaçóes necessárias. O desenvolvimento do SAF ocorreu a partir da identificaçáo da necessidade de se ter um modelo de registro de avaliaçáo conciso, com uso eficiente do tempo e que fosse facilmente aplicado a uma clientela culturalmente diversa e com dificuldades biopsicossociais variadas. Para sua construção foi eleito o Modelo de Ocupação Humana como referência, mas também as Diretrizes para Terapia Ocupacional Centrada no Cliente. Além disso, os terapeutas ocupacionais envolvidos buscaram o feedback direto com a equipe de atendimento sobre compreensão, clareza e frequência de uso do modelo proposto, para realizar as revisões necessárias. O formato do SAF da versão até o momento da publicação foi composto por duas partes: a primeira 
é descritiva e contém comentários gerais, lista de problemas e objetivos do cliente e plano de tratamento; a segunda é constituída por um checklist com as habilidades essenciais avaliadas pelo terapeuta ocupacional, com espaço para comentários. Também são apontados os benefícios verificados com o uso do modelo proposto, sem descrever a metodologia para obtenção desses dados, observando-se que, nesse formato, o SAF mostrou-se eficiente para verificar o progresso do cliente ao longo do tempo e que também auxiliou na identificação do papel e escopo do terapeuta ocupacional para o leitor.

Mais recentemente, Bart et al. (2011) apresentaram um modelo para documentar a intervenção terapêutica ocupacional de uma única sessão, referente ao processo de intervenção, o Documentation of Occupational Therapy Session during Intervention (DOTSI). Para seu desenvolvimento foi utilizado como referencial teórico a Estrutura da Prática da Terapia Ocupacional: Domínio e Processo, da AOTA, e a Classificaçáo Internacional de Funcionalidade, Incapacidade e Saúde - CIF, da Organização Mundial de Saúde. O DOTSI deve ser preenchido ao final da intervenção, informando o nome de cada atividade realizada e o tempo de duração, e para cada uma segue-se uma parte que inclui oito aspectos: contexto físico do tratamento, contexto social do tratamento, tipos de intervenção, fatores do cliente, habilidades de desempenho, padrôes de desempenho, áreas de ocupação e estratégias de intervenção. Também há um local para serem descritas as respostas da criança durante cada atividade. Para sua validaçáo participaram do estudo 55 terapeutas ocupacionais que atuavam com crianças, que documentaram duas a três sessões de atendimento. No total, 120 sessōes foram registradas. A validade de construto foi avaliada através das diferenças entre grupos conhecidos, com base em grupos de idade (pré-escolares e escolares) e com base em grupos de contexto (clínico ou educacional). Como resultado obteve-se significativa confiabilidade interavaliadores na maioria das subcategorias e a validade de construto foi estabelecida por diferenças significativas entre as duas configuraçôes (educacional e clínica) e os dois grupos etários. Dessa forma, verificou-se que o DOTSI demonstrou propriedades psicométricas aceitáveis, podendo ser usado como uma medida confiável e válida para a documentação das intervençôes terapêuticas ocupacionais. Seu uso pode estimular o desenvolvimento do raciocínio clínico pelo terapeuta ocupacional e o modelo pode ser adaptado para o uso em outros contextos e com outras populações que não a pediátrica clínica e educacional, assim como por outros profissionais da saúde.

Em todos os quatro estudos identificados é enfatizada a necessidade de desenvolvimento de modelos específicos de registro de intervenções de Terapia Ocupacional a partir de referenciais teóricos bem delimitados, associados à contextualização em relação ao público alvo a quem os registros se destinam e à populaçáo e contexto de atendimento terapêutico. Todos apontam que o uso dos modelos de registro favorece a função da documentação clínica de apoio à intervençâo clínica, além de proporcionar maior objetividade e registro da especificidade de atuação da Terapia Ocupacional.

Observam-se nos quatro estudos outras preocupaçóes em comum para o desenvolvimento e uso de modelos específicos de registro, incluindo-se diminuição do tempo gasto para sua execução, uso de linguagem técnica específica da profissão e direcionamento das informaçóes a serem registradas, de forma a favorecer a objetividade do conteúdo, produzindo as especificidades da Terapia Ocupacional e possibilitando mensurar e comparar os resultados de intervenção.

Nos três primeiros estudos nota-se um foco maior no fornecimento de orientações sobre o tipo de informação a ser registrada e a associação entre ela e os modelos teóricos adotados, e apresentam-se os benefícios de seu uso de forma subjetiva. Já no último, o foco maior é nos aspectos mais objetivos dos registros, como a possibilidade de mensuração e comparaçáo ao longo do tempo e entre atendimentos realizados junto a outros clientes/pacientes/usuários, e é realizado um estudo metodologicamente estruturado para desenvolvimento e validaçáo do formulário apresentado.

\subsection{Percepção e atitudes dos profissionais}

Pierre e Sonn (1999) referem que as percepções e interpretaçóes de eventos profissionais, incluindo os registros clínicos, podem sofrer influência de motivos inconscientes decorrentes de pensamentos, ideias ou emoçóes. Em diversos artigos analisados foi possível verificar aspectos referentes às percepçóes e atitudes dos terapeutas ocupacionais em relação à prática da documentaçáo clínica.

Bailey (1990) realizou um estudo com 696 terapeutas ocupacionais que abandonaram a carreira, com o objetivo de prevenir desgastes e trazer de volta os terapeutas ocupacionais, como um meio de resolver o problema de escassez de pessoal 
vivenciado no contexto onde tal estudo inseria-se. Dificuldades percebidas relacionadas à documentaçáo envolvida com a atuação profissional, referida como papelada, foi o terceiro aspecto mais apontado pelos entrevistados como aquele de que menos gostavam no trabalho e que contribuiu para que deixassem a carreira de terapeuta ocupacional. No entanto, a autora não discute as implicações dessa resposta.

Fearing (1991) referiu que a prática da documentação clínica é frequentemente mencionada pelos terapeutas ocupacionais como uma barreira para a satisfação com o trabalho, o que pode levar inclusive ao abandono da profissáo. Pontuou que se deve dar atenção aos problemas experimentados pelos profissionais na prática clínica, sendo importante identificar as causas de insatisfação. Afirma que os terapeutas ocupacionais devem assumir a responsabilidade pelo uso de métodos de resoluçáo de problemas, para lidarem com as dificuldades decorrentes da prática da documentação e desenvolverem uma atitude mais positiva em busca da satisfação com a realização dos registros. Sugeriu um método que envolve a definição do problema, pontuando os diferentes aspectos que contribuem para que a prática da documentação clínica seja percebida como problemática; a definição da expectativa esperada com os seus registros clínicos; e o desenvolvimento de um plano que possibilite seu alcance, resolvendo os problemas identificados.

Pierre e Sonn (1999) apresentaram um estudo realizado na Suécia com 11 terapeutas ocupacionais que atuavam em serviço especializado em idosos, com objetivo de identificar os significados associados ao conceito de documentação própria da Terapia Ocupacional. Cinco diferentes contradiçóes foram percebidas pelos profissionais dentro do processo de realização da documentação: entre a linguagem cotidiana e a linguagem profissional, entre a documentação altamente estruturada e a comunicação de uma visão global do paciente, entre as demandas jurídicas e éticas e as condiçôes ambientais de trabalho, entre diferentes expectativas dos vários destinatários dos registros e entre o raciocínio clínico terapêutico ocupacional e médico. Concluíram que, apesar do número pequeno da amostra, o estudo fornece informaçóes sobre preocupaçóes dos terapeutas ocupacionais referentes à documentação, enfatizando a importância de esclarecer a linguagem técnica profissional.

Posteriormente, Pierre (2001) publicou outra parte do mesmo estudo, com o objetivo de verificar quais práticas os terapeutas ocupacionais valorizam em seu dia a dia de trabalho, mas não incluem na documentação clínica. Para isso entrevistaram os 11 profissionais e analisaram o conteúdo de 22 prontuários, com base no Código de Ética e em outros documentos locais sobre documentação clínica e Terapia Ocupacional. Identificaram que mesmo quando os registros eram realizados de acordo com o processo terapêutico estabelecido, os profissionais podiam se sentir insatisfeitos com eles, por motivos que iam além dos aspectos ético-legais. Observaram que algumas práticas eram valorizadas e realizadas porém não registradas, como: focar no desempenho ocupacional do paciente; estabelecer contato e diálogo diário com os pacientes em situações não tidas como de atendimento propriamente dito; supervisionar náo formalmente o paciente nas atividades diárias, obtendo informaçóes que serão utilizadas para o processo terapêutico; oferecer apoio emocional e compreender os problemas do paciente. Tais açóes foram identificadas como domínios não registrados, principalmente por questốes relacionadas à linguagem técnica, apontando grande dificuldade dos terapeutas ocupacionais em nomeá-las. Finalizaram apontando que o próximo grande desafio da profissão seria o estabelecimento de uma "linguagem da ocupaçáo".

Davis et al. (2008) realizaram um estudo para verificar as opinióes, percepçóes e atitudes de terapeutas ocupacionais e de outros profissionais envolvidos com a assistência sobre a comunicação de evidências na documentação clínica, com base no modelo da Prática Baseada em Evidência (PBE). Para isso foi aplicado questionário on-line com 126 terapeutas ocupacionais clínicos do centro-oeste dos Estados Unidos e empregada metodologia de pesquisa qualitativa, pesquisa Delphi, com 11 outros profissionais (gestores e integrantes de agências financiadoras). Observaram que os profissionais apontam barreiras para a atuaçáo segundo a PBE, tais como restrição de tempo, exigência de produtividade e falta de entendimento das pesquisas publicadas. Verificaram ainda que essas barreiras também interferem na documentação, nos registros dos pacientes, das evidências utilizadas para embasar a intervenção. Além disso, os profissionais consideram necessário comunicar as evidências que embasam suas intervençóes quando precisam justificar financiamento do serviço prestado ou quando o serviço/instituição de trabalho o exige. Observaram ainda que comunicar evidências nos registros dos pacientes é uma prática inovadora, que não existem modelos ou diretrizes para sua execução e que seriam necessárias iniciativas organizacionais para dar suporte a essa ação.

Quanto à percepção e atitudes dos profissionais, foi verificada nas diferentes publicaçōes ênfase em aspectos negativos em relação à prática da 
documentação clínica, que podem levar ao não registro de algumas intervençóes e até mesmo ao abandono da carreira. No entanto, não foram explorados os aspectos positivos que podem estar associados ao desenvolvimento dessa atividade.

De forma geral verificou-se que a prática da documentação clínica pode ser percebida pelos terapeutas ocupacionais como geradora de insatisfação profissional (BAILEY, 1990; FEARING, 1991), o que pode gerar dilemas oriundos de contradições existentes na sua execução (PIERRE; SONN, 1999), especialmente no que se refere à linguagem apropriada a ser utilizada, podendo inclusive levar ao não registro de intervenções realizadas pela dificuldade percebida em descrevê-las de forma aceita como satisfatória (PIERRE, 2001).

Além disso, foram identificados alguns fatores que podem influenciar a percepção e atitudes dos terapeutas ocupacionais em relação aos registros clínicos, como necessidade de fornecer justificativas para o financiamento do serviço prestado e exigências institucionais.

\subsection{Comunicação e linguagem}

Uma das finalidades primordiais da documentação clínica é dar apoio à assistência prestada, servindo como um importante meio de comunicação entre todos os envolvidos (FEARING, 1993).

Nesse sentido, os diferentes artigos anteriormente citados que abordam modelos específicos de registro de intervenções terapêuticas ocupacionais informam que o uso de tais modelos favorece uma melhor comunicação, podendo, entre outras coisas, facilitar o entendimento da equipe e de demais leitores sobre os objetivos e papel da Terapia Ocupacional (FEARING, 1993; KYLE; WRIGHT, 1996) e agilizar possíveis encaminhamentos indicados ao cliente/paciente/usuário (WATSON, 1992).

Por se tratar de uma forma de comunicaçáo, a documentação clínica precisa utilizar uma linguagem correta, com terminologia apropriada para o ambiente e pertinente ao terapeuta ocupacional (PERINCHIEF, 2002).

Como já apontado nos estudos de Pierre e Sonn (1999) e Pierre (2001), a linguagem técnica foi referida pelos terapeutas ocupacionais investigados como um importante problema na realização da documentação clínica. Os problemas referentes à linguagem mostraram-se relacionados principalmente à dificuldade de descrever os aspectos mais específicos do escopo da Terapia Ocupacional no que diz respeito às atividades e ocupações, inferindo-se que os termos utilizados para descrevê-los muitas vezes são simples, do senso comum. Isso foi apontado como positivo para a facilitação da leitura e entendimento pelos pacientes/clientes/usuários, no entanto muitas açôes não são registradas pelos terapeutas ocupacionais por eles não terem certeza de que termos utilizar e por a descrição dessas açôes poder ser considerada inadequada para um registro clínico, não assemelhando-se a uma linguagem técnica profissional.

Tais estudos foram realizados anteriormente à publicação de iniciativas encontradas na literatura com o propósito de proporcionar uma terminologia específica da Terapia Ocupacional, sendo possível que a percepçáo dos profissionais se modifique a partir da divulgação e apropriação dessas iniciativas.

Duas propostas sobre linguagem comum para a Terapia Ocupacional foram localizadas na literatura técnico-científica. A primeira, da AOTA, é constituída por diversos documentos desenvolvidos a partir do foco da profissão no estudo da ocupação, na tentativa de delinear uma linguagem comum dos constructos que sustentam a Terapia Ocupacional, independente da área de atuação (DRUMMOND, 2007). Eles são considerados referência, principalmente no país de origem, para que o profissional possa comunicar com mais facilidade o seu trabalho em equipes, em eventos e publicaçóes e para esclarecer como se aplicam os conhecimentos específicos da Terapia Ocupacional (DRUMMOND, 2007). A segunda, da Rede Europeia de Terapia Ocupacional no Ensino Superior, é fruto de um projeto que teve por propósito auxiliar na harmonização da educaçáo em Terapia Ocupacional na Europa. O projeto culminou com a publicaçáo de um livro, no qual se apresenta uma estrutura conceitual, construída a partir de termos chaves usados pelos terapeutas ocupacionais para descreverem e explicarem sua prática (CREEK, 2010). Ambas as propostas foram desenvolvidas sob a influência do novo modelo de saúde proposto pela CIF (AMERICAN..., 2008b; CREEK, 2010).

Em relação à CIF, Darzins, Fone e Darzins (2006) referem que a estrutura conceitual e o vocabulário por ela apresentado pode promover a melhor documentação e comunicação pelos terapeutas ocupacionais e demais profissionais das equipes de saúde. Ressaltam que os terapeutas ocupacionais apresentam mais facilidade para entender os conceitos da CIF devido ao papel central da ocupação em sua proposta, mas que sua terminologia não deve substituir a linguagem específica da Terapia Ocupacional e sim coexistir e ser empregada conforme conveniência. 
A partir de tais informaçóes, pode-se considerar que o desenvolvimento de uma linguagem específica da profissão Terapia Ocupacional se apresenta como uma necessidade percebida pelos próprios profissionais. Pierre e Sonn (1999) questionam se os terapeutas ocupacionais almejariam uma linguagem específica na tentativa de terem mais reconhecimento e respeito diante da equipe e, dessa forma, terem a expectativa de uso de termos mais sofisticados e complicados, buscando aparentar mais cientificidade. No entanto, apontam que tal iniciativa poderia alienar ainda mais as pessoas, em especial os clientes/pacientes/usuários e seus familiares. Ressaltam ainda que se a linguagem será utilizada na documentação clínica, por mais que ela traga especificidades da profissão, é importante que seja de fácil compreensão pelos diferentes leitores, para que a comunicaçáo se efetive.

Também se observa, a partir dos apontamentos nos diferentes artigos, ser adequada a configuração de uma linguagem que proporcione a descrição do escopo de atuação da Terapia Ocupacional, desenvolvida a partir dos referenciais teóricos adotados para a atuação. Nesse sentido, Kyle e Wright (1996) destacam que o uso de referencial teórico é importante para a realização da documentação clínica, mas que seu papel é maior em relaçấo ao conteúdo, podendo a terminologia adotada ser adaptada para melhorar o entendimento pelo público a quem o registro se destina. Fearing (1993) ainda ressalta que, para utilizaçấo de uma linguagem mais apropriada ao terapeuta ocupacional, é mais indicada a descrição dos comportamentos ao invés do uso de termos que tragam rótulos predefinidos, que podem levar a entendimentos diferentes de acordo com quem escreve.

\subsection{Qualidade}

Outro aspecto implicado com a documentação clínica é a qualidade apresentada nos registros, sendo que Hedberg-Kristensson e Iwarsson (2003) ressaltam que a documentaçáo realizada de forma incorreta pode influenciar negativamente o tratamento terapêutico ocupacional. Já Darzins, Fone e Darzins (2006) afirmam que as práticas de registro muitas vezes podem ser ineficientes por não mostrarem o que é realmente importante para o cliente e os benefícios da intervenção, o que pode levar a uma desvalorização do processo de reabilitação em geral e da Terapia Ocupacional em particular. Em artigos da presente revisão, alguns aspectos relacionados à qualidade da documentaçáo clínica dos terapeutas ocupacionais foram abordados.
McGuire (1997), ao tratar da documentação clínica no atendimento domiciliar de Terapia Ocupacional, busca clarificar alguns princípios que os profissionais podem utilizar para incrementar a eficiência e o potencial para o reembolso dos serviços prestados. No entanto, busca ampliar a aplicação de sua proposta, ao afirmar que tais princípios podem ser utilizados de forma geral na documentação clínica de Terapia Ocupacional, para que ela tenha um padrão excelente, destacando: a necessidade de se concentrar nos aspectos da funcionalidade do cliente, envolvendo os níveis prévios e atuais e a evolução da função em relação aos objetivos do tratamento; explicitação das causas que restringem a função; informação do progresso, seus possíveis motivos de lentidão ou retrocesso, e das expectativas do tratamento; explicitar nível de segurança e competência para desempenho funcional; e dar maior enfoque na descrição dos tipos de serviços especializados oferecidos, ao invés de na descrição detalhada das atividades desenvolvidas nos atendimentos.

Hedberg-Kristensson e Iwarsson (2003) desenvolveram um estudo para avaliar aspectos relacionados à qualidade dos registros com foco particular no processo de prescrição de tecnologia assistiva. Ao todo, 182 registros, de serviços de dois municípios da Suécia com rotinas distintas, foram avaliados segundo um protocolo desenvolvido com base na legislação local, recomendaçóes oficiais de órgãos de saúde e de Terapia Ocupacional, e experiência prática em Terapia Ocupacional e prescrição de tecnologia assistiva. Os itens avaliados foram: dados administrativos, níveis de anamnese (ou avaliação), níveis de status (padrão funcional), níveis de análise (problemas identificados), níveis de objetivos, níveis de medidas durante acompanhamento e níveis de resultados. Identificaram que nenhum dos dois grupos demonstrou alto nível de qualidade, com falhas ou ausência de registros dos diferentes itens avaliados, e concluiu que é necessário o desenvolvimento de qualidade na documentação da Terapia Ocupacional, de forma geral e especificamente em relação à prescrição de tecnologia assistiva.

Também foi pontuado por Fearing (1993) que o uso do modelo apresentado em seu trabalho para registro das intervençôes terapêuticas ocupacionais, citado anteriormente, favorece a qualidade dos registros, produzindo informações consistentes e organizadas, independentemente do terapeuta ocupacional que a tenha desenvolvido. A autora refere que um modelo de registro, independente de qual seja, deve proporcionar que, para uma dada situação, dois ou mais terapeutas ocupacionais 
devem ser capazes de registrar informaçóes iguais ou equivalentes.

Simpson (1998) trouxe orientaçóes sobre a boa documentação para processos de auditoria de Fisioterapia e Terapia Ocupacional, com foco na reabilitação de pessoas idosas que caíram. Esse autor não se refere somente à documentação clínica como abordada neste estudo, mas sim a todos os possíveis documentos que facilitem a avaliação e escolha de intervenção de reabilitação para um determinado problema clínico e promovam um nível elevado de prática e ensino, como declaraçôes políticas locais, diretrizes, protocolos, listas de verificação, registros de pacientes e outros materiais impressos. No que diz respeito aos registros clínicos, de forma geral, algumas orientaçôes podem ser utilizadas, como: registrar todas as informaçôes pertinentes à assistência, pois se não está registrado, por melhor que seja o atendimento prestado, ele não será reconhecido como realizado; acrescentar identificação a todos os formulários utilizados para registro, incluindo local/departamento, título, número da versão e data de configuração; e registrar sempre que uma resposta for negativa, não deixando dúvidas sobre se um aspecto foi investigado ou náo junto ao cliente/ paciente/usuário.

Observou-se nos diferentes artigos que a qualidade dos registros realizados pelo terapeuta ocupacional pode influenciar o tratamento oferecido, o reconhecimento da profissão e a avaliação dos serviços em auditorias.

Apenas um estudo avaliando a qualidade dos registros dos terapeutas ocupacionais foi identificado, sendo apontada a baixa qualidade deles. No entanto, mostram-se ainda insipientes os dados sobre a qualidade da documentação clínica desenvolvida pelos terapeutas ocupacionais que revelem quais os pontos positivos e os aspectos a serem melhorados para sua realizaçáo.

Alguns princípios e orientaçôes para que registros de Terapia Ocupacional sejam realizados com melhor qualidade são identificados e o uso de um modelo para registro também demonstrou colaborar para a maior qualidade da documentação.

Além desses temas apresentados no presente estudo, outros diferentes temas estáo relacionados à documentação clínica e não foram abordados nos artigos desta revisão, ou sua abordagem ocorreu de forma superficial, como aspectos ético-legais, formatos de registros (manuais ou informatizados) e aspectos da formação profissional.

Por exemplo, quanto à formação dos terapeutas ocupacionais no tocante à documentação clínica, apenas Fearing (1993) faz uma referência, afirmando que apesar de o desenvolvimento dos registros clínicos ser uma responsabilidade profissional do terapeuta ocupacional, seu aprendizado é geralmente uma experiência desorganizada e, muitas vezes, insatisfatória para os profissionais.

Já em relação aos aspectos ético-legais, HedbergKristensson e Iwarsson (2003) relatam que utilizaram a legislação do local onde desenvolveram seu estudo como base para avaliar a qualidade dos registros, reforçando a importância do conhecimento das exigências e recomendaçóes legais para o desenvolvimento de documentação clínica com qualidade.

\section{Considerações finais}

No presente estudo foram identificados artigos que possibilitaram uma primeira aproximação com o tema e uma discussão inicial sobre diferentes aspectos envolvidos com a documentaçáo clínica em Terapia Ocupacional.

Foi verificado que mais artigos triados apresentavam, pela leitura dos resumos, possibilidades de atendimento aos critérios de inclusão, porém foram encontradas dificuldades para acesso ao texto deles na íntegra, como custo elevado para aquisição e ausência de exemplares disponíveis nas bibliotecas consultadas. Também se mostrou como limitadora a língua de publicação, sendo incluídos neste estudo apenas os textos publicados em língua inglesa ou espanhola, indicando a necessidade de novas pesquisas e busca de acesso a publicações em outras línguas, em trabalhos futuros.

Além disso, nenhum dos artigos triados era nacional, indicando a necessidade de maior desenvolvimento do tema pelos terapeutas ocupacionais brasileiros. No entanto, ao longo do estudo ocorreu a publicação de um capítulo de livro e da Resoluçáo COFFITO 415/2012, ambos sobre documentação clínica da Terapia Ocupacional, demonstrando a atualidade do tema e a conquista de espaço para sua discussão entre os profissionais.

A partir das informações obtidas foi possível identificar alguns aspectos envolvidos com a documentação clínica em Terapia Ocupacional. No entanto, nota-se que as informaçôes são bastante segmentadas e as pesquisas, pouco comparáveis entre si, sugerindo diversas lacunas que merecem maior atenção e o desenvolvimento de novos estudos para que se forme um corpo de conhecimento mais consistente para a melhor prática da documentação clínica em Terapia Ocupacional. 
Dentre as possibilidades de futuras pesquisas brasileiras, verificou-se a necessidade de investigar a percepção dos profissionais sobre o desenvolvimento dos próprios registros, identificando o que percebem de positivo e negativo nesta prática, assim como verificando como se dá o desenvolvimento dos registros por eles e quais são os parâmetros éticolegais instituídos. Tais questóes foram abordadas no projeto de mestrado intitulado "A documentação clínica ambulatorial sob a óptica de terapeutas ocupacionais", para o qual esta revisão serviu de embasamento.

Além desses, vários outros aspectos podem ser aprofundados em futuras investigaçóes, como, por exemplo: a qualidade dos registros desenvolvidos; a efetividade da comunicação, tanto para os próprios terapeutas ocupacionais como para os diferentes integrantes das equipes e, em especial, para o paciente/ cliente/usuário e seus familiares/responsáveis; o uso de modelos de registro, identificando como e por quem foram elaborados, os referenciais teóricos que embasaram sua construção e as vantagens e desvantagens de seu uso; os aspectos da formação profissional sobre a documentação clínica; e as implicaçôes éticas relacionadas ao desenvolvimento dos registros clínicos.

Destacou-se, nas diferentes publicaçôes, o impacto que o modelo teórico adotado para embasar a atuação clínica apresenta na prática dos registros pelos terapeutas ocupacionais, tanto com relação ao tipo de informação a constar e/ou estruturação do modelo de registro, como com relação à linguagem utilizada. Este trabalho abre, portanto, uma possibilidade de novos focos de pesquisa sobre documentação clínica em Terapia Ocupacional.

\section{Referências}

AMERICAN OCCUPATIONAL THERAPY ASSOCIATION - AOTA. Guidelines for Documentation of Occupational Therapy. American Journal of Occupational Therapy, Bethesda, v. 62, n. 6, p. 684-690, Nov/Dec 2008a. http://dx.doi.org/10.5014/ajot.62.6.684

AMERICAN OCCUPATIONAL THERAPY ASSOCIATION - AOTA. Occupational Therapy Practice Framework: Domain and Process. 2nd ed. (Framework-II). American Journal of Occupational Therapy, Bethesda, v. 62, n. 6, p. 625-683, nov./dec. 2008 b. http://dx.doi. org/10.5014/ajot.62.6.625

BART, O. et al. Development and validation of the Documentation of Occupational Therapy Session during Intervention (D.O.T.S.I.). Research in Developmental Disabilities, Baton Rouge, v. 32, n. 2, p. 719-726, 2011. PMid:21134729. http://dx.doi.org/10.1016/j. ridd.2010.11.008
BAILEY, D. M. Reasons for Attrition From Occupational Therapy. American Journal of Occupational Therapy, Bethesda, v. 44, n. 1, p. 23-29, Jan 1990. http://dx.doi. org/10.5014/ajot.44.1.23

\section{CONSELHO FEDERAL DE FISIOTERAPIA E} TERAPIA OCUPACIONAL - COFFITO. Resolução COFFITO no 415, de 19 de maio de 2012. Dispóe sobre a obrigatoriedade do registro em prontuário pelo terapeuta ocupacional, da guarda e do seu descarte e dá outras providências. Diário Oficial da República Federativa do Brasil, Brasília, DF, 23 maio 2012. Seção 1.

CREEK, J. The Core Concepts of Occupational Therapy: a Dynamic Framework for Practice. London: Jessica Kingsley Publishers, 2010.

DAVIS, J. et al. Communicating evidence in clinical documentation. Australian Occupational Therapy Journal, Melbourne, v. 55, n. 4, p. 249-255, 2008. PMid:20887477. http://dx.doi.org/10.1111/j.1440-1630.2007.00710.x

DARZINS, P.; FONE, S.; DARZINS, S. The International Classification of Functioning, Disability and Health can help to structure and evaluate therapy. Australian Occupational Therapy Journal, Melbourne, v. 53, n. 2, p. 127-131, 2006. http://dx.doi. org/10.1111/j.1440-1630.2006.00580.x

DRUMMOND, A. F. Fundamentos da Terapia ocupacional. In: CAVALCANTI, A.; GALVÃO, C. Terapia Ocupacional: Fundamentaçáo e Prática. Rio de Janeiro: Guanabara Koogan, 2007. cap. 2, p. 10-17.

FEARING, V. G. Managing paperwork: problem solving for job satisfaction. Canadian Journal of Occupational Therapy, Otawwa, v. 58, n. 2, p. 55-57, 1991. http:// dx.doi.org/10.1177/000841749105800201

FEARING, V. G. Occupational therapists chart a course through the health record. Canadian Journal of Occupational Therapy, Toronto, v. 60, n. 5, p. 232-240, 1993. http:// dx.doi.org/10.1177/000841749306000504

GRANT, M. J.; BOOTHT, A. A typology of reviews: an analysis of 14 review types and associated methodologies. Health Information and Libraries Journal, Oxford, v. 26, n. 2, p. 91-108, 2009. PMid:19490148. http://dx.doi. org/10.1111/j.1471-1842.2009.00848.x

HEDBERG-KRISTENSSON, E.; IWARSSON, S. Documentation Quality in Occupational Therapy Patient Records: Focusing on the Technical Aid Prescription Process. Scandinavian Journal of Occupational Therapy, Oslo, v. 10, p. 72-80, 2003. http://dx.doi. org/10.1080/11038120310009434

KYLE, T.; WRIGHT, S. Reflecting the Model of Human Occupation in occupational therapy documentation. Canadian Journal of Occupational Therapy, Toronto, v. 63, n. 3, p. 192-196, 1996. http://dx.doi. org/10.1177/000841749606300306

MASSAD, E.; MARIN, H. F.; AZEVEDO NETO, R. S. Prontuário eletrônico do paciente: definiçóes e conceitos. In: MASSAD, E.; MARIN, H. F.; AZEVEDO NETO, R. S. (Ed.). O prontuário eletrônico do paciente na assistência, informação e conhecimento médico. São Paulo: H. de F. Marin, 2003. cap. 1, p. 1-15. 
MATTHEWS, M. M.; JABRI, J. L. Documentação dos Serviços de Terapia Ocupacional. In: PEDRETTI, L. W.; EARLY, M. B. Terapia ocupacional: capacidades práticas para disfunçôes físicas. Tradução de Lúcia Speed Ferreira de Mello, Cláudio Assencio Rocha; Revisão científica de Angela Gonçalves Marx. São Paulo: Roca, 2004. cap. 10 , p. $98-107$.

McGUIRE, M. J. Documenting progress in home care. American Journal of Occupational Therapy, Bethesda, v. 51, n. 6, p. 436-445, Jun 1997. http://dx.doi.org/10.5014/ ajot.51.6.436

MEZZOMO, A. A. Introdução. In: MEZZOMO, A. A. Serviço do prontuário do paciente: organização e técnica. 4. ed. São Paulo: Centro São Camilo de Desenvolvimento em Administração da Saúde, 1991. cap. 1, p. 7-30.

NOVAES, H. M. D. A evolução do registro médico. In: NOVAES, H. M. D. et al. (Ed.). O prontuário eletrônico do paciente na assistência, informação e conhecimento médico. Sáo Paulo: H. de F. Marin, 2003. cap. 3, p. 39-46.

OLIVEIRA, A. S. et al. Terapia Ocupacional. In: GAlvãO, M. C. B.; RICARTE, I. L. M. Prontuário do Paciente. Rio de Janeiro: Guanabara Koogan, 2012. cap. 15 , p. $207-221$.

PERINCHIEF, J. M. Controle dos Serviços de Terapia Ocupacional. In: NEISTADT, M. E.; CREPEAU, E. B. Willard \& Spackman Terapia Ocupacional. Tradução de Cláudia Lúcia Caetano de Araújo et al. Revisão técnica de Eliane Ferreira. 9. ed. Rio de Janeiro: Guanabara Koogan, 2002. cap. 43, p. 722-738.

PIERRE, B. L.; SONN, U. Occupational therapy as documented in patient's records - Part II: What is proper documentation? Contradictions and aspects of concern from the perspective of OTs. Scandinavian Journal of Occupational Therapy, Oslo, v. 6, n. 1, p. 3-10, 1999. http://dx.doi.org/10.1080/110381299443799

PIERRE, B. L. Occupational therapy as documented in patient's records - Part III: Valued but not documented. Underground practice in the context of professional written communication. Scandinavian Journal of Occupational Therapy, Oslo, v. 8, n. 4, p. 174-183, 2001. http://dx.doi. org/10.1080/110381201317166531

POSSARI, J. F. Prontuário do Paciente. In: POSSARI, J. F. Prontuário do paciente e os registros de enfermagem. 2. ed. São Paulo: Iátria, 2007. cap. 1, p. 17-34.
RADOMSKI, M. V. Planejamento, orientação e documentação da terapia. In: TROMBLY, C.A.; RADOMSKI, M.V. Terapia Ocupacional para Disfunçōes Físicas. Tradução e revisão científica de Beatriz Bittencourt Granjo Schlecht et al. 5. ed. São Paulo: Livraria Santos Editora, 2005. cap. 19, p. 443-461.

REIS, A. O. A. et al. Prontuários, para quem servem? Representação dos coordenadores de equipe dos CAPSi a respeito do valor e utilidade dos prontuários. Revista Brasileira de Crescimento e Desenvolvimento Humano, São Paulo, v. 19, n. 3, p. 383-392, 2009.

ROGERS, J. C.; HOLM, M. B. Occupational Therapy diagnostic reasoning: A component of clinical reasoning. American Journal of Occupational Therapy, Bethesda, v. 45, n. 11, p. 1045-1053, 1991. http://dx.doi.org/10.5014/ ajot.45.11.1045

SAMES, K. M. Use of Language. In: SAMES, K. M. Documenting occupational therapy practice. New Jersey: Pearson, 2005. section 1, p. 3-36.

SIMPSON, J. M. How good is your documentation? Pyisiotherapy, United Kingdom, v. 84, n. 10, p. 469-471, Oct 1998.

SOARES, L. B. T. História da Terapia ocupacional. In: CAVALCANTI, A.; GALVÃO, C. Terapia Ocupacional: Fundamentação e Prática. Rio de Janeiro: Guanabara Koogan, 2007. cap. 1, p. 3-9.

STUMPF, M. K.; FREITAS, H. M. R. A gestão da informação em um hospital universitário: o processo de definição do Patient Core Record. Revista de Administraçâo Contemporânea, Rio de Janeiro, v. 1, n. 1, p. 71-99, abr. 1997. http://dx.doi.org/10.1590/ S1415-65551997000100005

VASCONCELLOS, M. M.; GRIBEL, E. B.; MORAES, I. H. S. Registros em saúde: avaliação da qualidade do prontuário do paciente na atenção básica, Rio de Janeiro, Brasil. Cadernos de Saúde Pública, Rio de Janeiro, v. 24, p. S173-S182, 2008. Suplemento 1. http://dx.doi. org/10.1590/S0102-311X2008001300021

WATSON, D. Documentation of paediatric assessments using the occupational therapy guidelines for client-centred practice. Canadian Journal of Occupational Therapy, Toronto, v. 59, n. 2, p. 87-94, 1992. http://dx.doi. org/10.1177/000841749205900204

\section{Contribuição dos Autores}

Carla Simon Benevides Panzeri: concepção e redação do texto manuscrito, organização e análise dos dados. Marina Silveira Palhares: concepção e redação do texto manuscrito. 